\title{
Solidons and rotons in liquid helium
}

\author{
M. Héritier, G. Montambaux and P. Lederer \\ Laboratoire de Physique des Solides (*), Université de Paris-Sud, Centre d'Orsay, 91405 Orsay, France \\ (Reçu le 21 juin 1979, révisé le 16 juillet 1979, accepté le 24 juillet 1979)
}

\begin{abstract}
Résumé. - La courbe de dispersion de phonon d'un liquide presque solide doit présenter un minimum de type roton, correspondant à un mouvement de translation d'atomes dans une petite région solide du liquide. Ce solidon, excitation élémentaire de $1^{14} \mathrm{He}$ super fluide ou fluctuation dans ${ }^{3} \mathrm{He}$, fournit une description satisfaisante du roton.
\end{abstract}

\begin{abstract}
The phonon dispersion curve of a nearly solid liquid is shown to present a roton-like minimum, which corresponds to a translational motion of atoms in a small solid region of the liquid. This solidon, which should exist as an elementary excitation in superfluid ${ }^{4} \mathrm{He}$ and as a fluctuation in ${ }^{3} \mathrm{He}$, gives a satisfactory description of a roton.
\end{abstract}

1. Introduction. - Describing the atomic motion of liquid Helium II in terms of elementary excitations has proved to be a very powerful method to interpret its superfluids properties [1]. However, Landau's assumptions about the existence of an energy gap for the individual excitations and about the phononroton dispersion curve were introduced empirically rather than on the basis of rigorous quantum hydrodynamics. In particular, his interpretation of a roton as a quantum of rotational motion was proved to be erroneous [2]. Still now, it is difficult to give a simple description of the atomic motions involved in a roton. Some authors have presented it as essentially one ${ }^{4} \mathrm{He}$ atom travelling rapidly through the other atoms which move out of its path [3]. However, the best description of a roton has been given by Feynman [4] and Feynman and Cohen [5]. They proposed a variational wave function of the excited state which accounts qualitatively for the observed roton spectrum. In their model, a roton can be pictured roughly as a vortex ring of such small radius that only one atom can pass through the centre. Usually, the concept of roton is restricted to the superfluid state of liquid ${ }^{4} \mathrm{He}$. However, we show in this letter that a roton-like minimum in the collective excitation dispersion curve can be encountered in other quantum liquids provided they are nearly solid (in a sense which will be precised later). The corresponding excitation is interpreted as a translational motion of atoms in a small solid region in

(*) Associé au C.N.R.S. the liquid. We call it a solidon. In principle, a solidon could exist in a non superfluid quantum liquid, even in a normal Fermi liquid. However, in most cases, it would be strongly scattered by individual excitations, and then would constitue a solid fluctuation or a parasolidon rather than a well-defined elementary excitation. According to us, liquid ${ }^{4} \mathrm{He}$ and liquid ${ }^{3} \mathrm{He}$ are the best examples of what we call a nearly solid liquid. Therefore, we suggest that the solidon is the right description of a roton. In fact, we believe that the Feynman and Cohen wave function is merely an approximate solidon wave function, essentially valid at large distances. This picture provides a very simple description of the roton and a transparent explanation of the minimum in the dispersion curve, as will be discussed below. We also predict the existence of parasolidons or pararotons in liquid ${ }^{3} \mathrm{He}$, which might have been observed recently [6].

2. Description of the solidon. - What do we call a nearly solid liquid ? Usually, in a classical liquid, a typical longitudinal phonon energy $k_{\mathbf{B}} \theta_{\mathbf{D}}$ (for wave vectors about $\pi / a$ ) is not large compared to the atomic kinetic energy which is of the order of the temperature of the liquid

$$
k_{\mathrm{B}} \theta_{\mathrm{D}} \ll k_{\mathrm{B}} T
$$

where $\theta_{\mathbf{D}}$ is the Debye temperature of the solid.

On the contrary, in quantum liquids, at lower temperatures, the zero-point atomic kinetic energy is of order $t=\hbar^{2} / 2 m a^{2}$ where $\dot{m}$ is the atomic mass and 
$a$ is the mean interatomic distance. As recently outlined by Castaing and Nozières [7], in liquid ${ }^{3} \mathrm{He}$ near its melting curve

$$
k_{\mathrm{B}} \theta_{\mathrm{D}} \gg t .
$$

This inequality reflects the nearly solid character of liquid ${ }^{3} \mathrm{He}$. The liquid is locally very rigid because of the high vibration energy $k_{\mathrm{B}} \theta_{\mathrm{D}}$ of the atoms in their own cages. But, it still possess the plasticity of a liquid because of the small energy $t$ to drift the cages around each other [7]. Morever, near enough to the melting curve, in any liquid,

$$
G_{\mathrm{s}}(p, T)-G_{1}(p, T) \ll t
$$

where $G_{\mathrm{s}}$ and $G_{1}$ are the free enthalpies per atom of respectively the solid phase and the liquid phase at a given pressure $p$ and a given temperature $T$. Eq. (2) defines a region of the phase diagram in the $(p, T)$ plane, more or less extended according to the value of $t$. The purpose of this letter is to study the consequences of these properties on the excitation spectrum of the liquid. We deal with nearly solid quantum liquids, namely liquids where inequalities (1) and (2) are satisfied. Of course, this is the case of liquid ${ }^{3} \mathrm{He}$, but also of liquid ${ }^{4} \mathrm{He}$, where the orders of magnitude are the same, near enough to the melting curve, and of their mixtures. We can consider $2 \mathrm{D}$ liquid layers as well as $3 \mathrm{D}$ bulk liquid.

In an ordinary liquid, i.e. where $\theta_{\mathrm{D}} \ll T$, the phonon dispersion curve increases monotonously. The larger the wave vector $\mathbf{q}$, the higher the phonon energy $\omega(\mathbf{q})$. On the contrary, in a solid, the phonon spectrum exhibits a periodicity :

$$
\omega(\mathbf{q})=\omega(\mathbf{q}+\mathbf{K})
$$

where $\mathbf{K}$ is a reciprocal lattice vector. This is a consequence of the translational invariance of the crystal. Let us consider a vibrational mode with wave vector $\mathbf{q}+\mathbf{K}$, in a perfect crystal, with an infinite number of atoms. It can always be considered as the superposition of a translational motion with momentum $\mathbf{p}$ and of a phonon with wave vector $\mathbf{q}$ and energy $\omega(\mathbf{q})$ but zero momentum. The atomic wave function in the crystal can be described by a superposition of plane waves, the wave vectors of which belong to the reciprocal lattice. Hence, the translation has a momentum $h \mathbf{K}$ and an energy $\hbar^{2} K^{2} / 2 \mathrm{Nm}$ in a finite crystal of $N$ atoms. The latter energy, i.e. the kinetic energy transferred to the lattice, vanishes in an infinite crystal. Therefore, the energies of acoustical phonons with wave vectors belonging to the reciprocal lattice vanish.

Some memory of this property should remain in the longitudinal phonon spectrum of a nearly solid liquid. At any point $(p, T)$ of the phase diagram, we can define a metastable crystal structure which minimizes $G_{\mathrm{s}}(p, T)$, the free enthalpy per atom in the solid state. Let us call $\mathbf{K}$ the reciprocal lattice vectors of this metastable crystal and $\mathbf{K}_{0}$, the shortest one. At least at $\mathbf{K}_{0}$, the phonon dispersion curve should exhibit a pronounced, although non-zero, minimum which should vanish at the liquid-solid transition, discontinuously in a first order transition, but continuously in a second order one. Obviously, the phonon energy at wave vector $\mathbf{K}_{0}$ can be lowered by forming a well ordered lattice. The larger $k_{\mathrm{B}} \theta_{\mathrm{D}}$, the more effective the lowering. This is obtained at the expense of a free enthalpy $G_{\mathrm{s}}(p, T)-G_{1}(p, T)$ per atom, which limits the number of atoms $N$ in this lattice to a finite value. Of course, the phonon energy of wave vector $\mathbf{K}_{0}$ does not vanish in this lattice of finite extension and increases when $N$ decreases. The balance between these two opposite effects determines the value of $N$ which minimizes the phonon energy. As stated above, the roton-like minimum occurs because the liquid is nearly solid (inequalities (1) and (2)). It is due to the formation of a local region in the liquid where the atoms are ordered as in a solid, in which the atomic motions involved in a phonon require much less energy than in the liquid. For that reason, we call it a solidon, inasmuch as it is a well-defined excitation with a long lifetime, and a parasolidon when its width becomes comparable to its energy.

Let us try to describe the structure of this solidon.

The solidon energy can be considered as the sum of two terms : the formation energy of this $N$-site lattice $E_{\text {lat }}(N, p, T)$ and the kinetic energy of the atomic motion $E_{\mathbf{k i n}}(N, p, T)$. We discuss, now, these two terms. When a solidon is formed, three regions of space should be, in principle, distinguished. In the first region, of radius $R$, the lattice is perfect or nearly perfect. In the second region, the order parameter decreases more or less smoothly over a length $1 / K$. The third region is completely liquid. The first region always exists, because $k_{\mathrm{B}} \theta_{\mathrm{D}}$ is much greater than any other energy in the problem. Any amount of disorder would increase appreciably the phonon energy. The physical value of $K R$ is not obvious. It may be small, near to the melting curve, if the solid-liquid surface tension is quite large, close to a first order transition, even though one could expect that a very gradual decay of the order is unfavourable. However, we approximate $E_{\text {lat }}$ by the sum of two terms :

$$
E_{1 \mathrm{lat}}(N, p, T)=N \delta G(p, T)+N^{2 / 3} \alpha
$$

In (3), $\delta G(p, T)=G^{*}(p, T)-G_{1}(p, T)$, where $G^{*}(p, T)$ is the free enthalpy per atom in the lattice, when boundary effects are ignored. $G^{*}(p, T)$ is lowered below $G_{\mathrm{s}}(p, T)$ by relaxation effects. The second term $N^{2 / 3} \propto$ represents the solid-liquid interface energy. In fact, this expression is essentially valid when $K R$ is large. When this is not true, one should add other terms with smaller powers of $N$. Such terms are not expected to be important when inequalities (1) and (2) are satisfied and we shall disregard them here. Of course, this surface term should be considered as 
phenomenological. In particular, $\alpha$ should not be confused with the macroscopic solid-liquid surface tension coefficient.

In this lattice of limited extension a low energy excitation with wave vector $K_{0}$ can be formed by transferring to the lattice a momentum $\hbar K_{0}$. The translation of the center of mass costs a kinetic energy

$$
E_{\mathbf{k i n}}^{1}=\frac{\hbar^{2} K_{0}^{2}}{2 N m}
$$

This term refers to the kinetic energy of the atoms inside the solid region. However, the particle current density does not stop suddenly at the solid-liquid interface. The current flow would not be conserved. We must consider a backflow in the disordered region. The current lines form loops schematized in figure 1 . If we assume that the only elementary excitations in the liquid are phonons with a dispersion relation

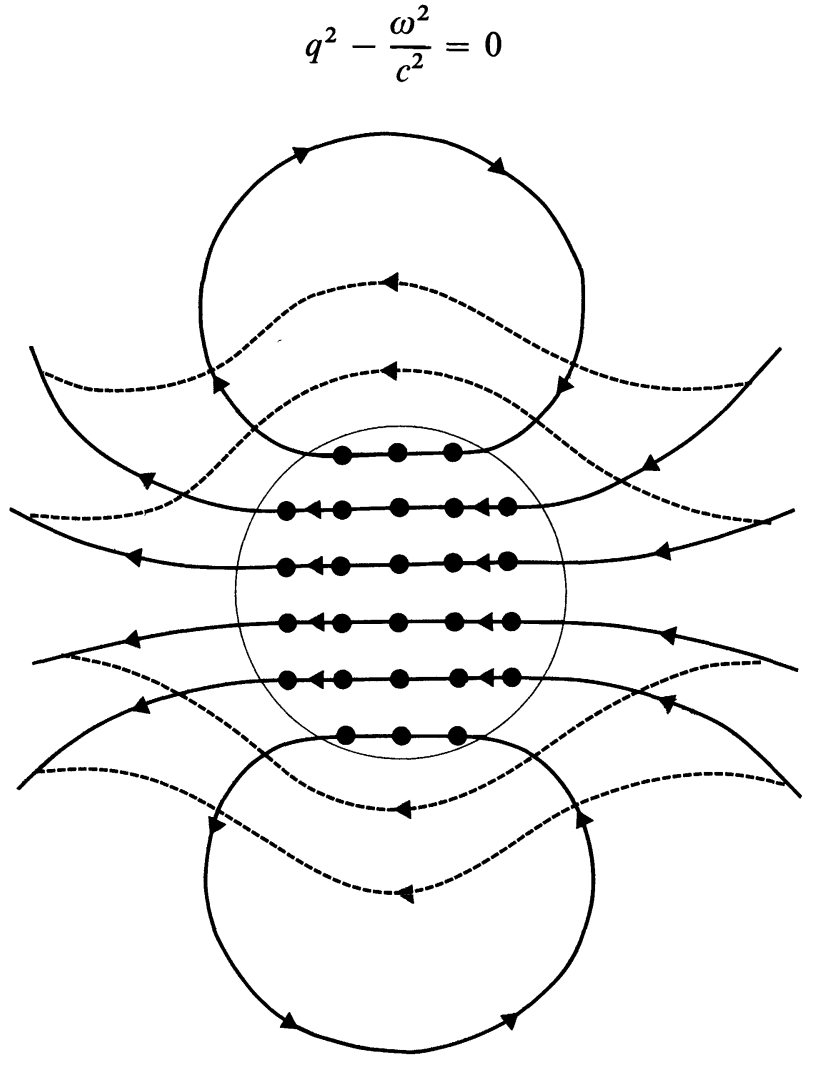

Fig. 1. - Schematic representation of a solidon. The atomic flow is shown by solid lines, the entropy flow by dotted line. The black dots are atoms.

an exact analogy can be made between the electric field in the vacuum and the velocity in the liquid. At large distances compared to $R$, the solidon can be considered as a source and a sink of atoms close together. The electrical analogue is a dipole. The blackflow kinetic energy in the liquid is equivalent to the electrostatic energy of a dipole :

$$
E_{\mathrm{kin}}^{2}=\frac{3 \pi a}{2} \frac{\hbar^{2} K_{0}^{2}}{2 N m}
$$

where $a$ is a geometrical factor, of order unity, which could be determined, in principle, by a variational procedure. The resultant of the backflow momentum outside of a sphere is zero. However, because of this backflow, the solidon can have a non-zero momentum $\hbar \mathbf{K}_{0}$, but a group velocity equal to zero when $p=\hbar \mathbf{K}_{0}$ [2]. We have ignored any possible effect of other solidons on the velocity field in the liquid and, therefore, of the solidon-solidon interaction.

We obtain an approximate expression for the solidon free enthalpy as a function of $N$ :

$$
\begin{aligned}
\Gamma(N, p, T)=\left(1+\frac{3 \pi a}{2}\right) & \frac{\hbar^{2} K_{0}^{2}}{2 N m}+ \\
& +N \delta G(p, T)+N^{2 / 3} \alpha
\end{aligned}
$$

which must be minimized with respect to $N$.

Two regimes are considered. In the first one, the last term in eq. (6), i.e. the surface term, is negligible compared to the volume term. Then, the solidon free enthalpy is

$$
\Gamma_{\mathrm{M}}(p, T)=2\left[\frac{\hbar^{2} K_{0}^{2}}{2 m}\left(1+\frac{3 \pi a}{2}\right) \delta G\right]^{1 / 2}
$$

corresponding to a number of atoms in the solid region

$$
N_{0}(p, T)=\left[\frac{\hbar^{2} K_{0}^{2}}{2 m}\left(1+\frac{3 \pi a}{2}\right) / \delta G\right]^{1 / 2} .
$$

As the liquid-solid equilibrium is approached, the number of atoms increases and the solidon free enthalpy decreases. However $N_{0}$ does not diverge and $\Gamma_{\mathrm{M}}$ does not go to zero at the transition because of the surface term.

In the second regime, the surface term is dominant in eq. (6), and we neglect the volume term. Then, the solidon free enthalpy is

$$
\Gamma_{\mathrm{M}}(p, T)=\frac{5}{2} \times\left(\frac{2}{3}\right)^{3 / 5}\left[\left(1+\frac{3 \pi a}{2}\right) \frac{\hbar^{2} K_{0}^{2}}{2 m}\right]^{2 / 5} \alpha^{3 / 5}
$$

corresponding to a number of atoms in the solid region :

$$
N_{0}(p, T)=\left[\frac{3}{2}\left(1+\frac{3 \pi a}{2}\right) \frac{\hbar^{2} K_{0}^{2}}{2 m} / \alpha\right]^{3 / 5} .
$$

This second regime is obtained in the vicinity of the melting curve. The cross-over between the two regimes occurs when the surface term and the volume term are equal. This defines a cross-over line in the $(p, T)$ plane, approximately given by

$$
\delta G(p, T) \simeq \alpha^{6 / 5}\left[\left(1+\frac{3 \pi a}{2}\right) \frac{\hbar^{2} K_{0}^{2}}{2 m}\right]^{-1 / 5} .
$$


It may happen that two crystal structures are nearly degenerate, but lead to quite different values of $\alpha$. In the first regime, the stable solidon structure will always be the lower energy one. In the second regime, i.e. near the melting curve, it will be governed by the lower value of $\alpha$, which might not correspond to the lower value of $\delta G$.

In what precedes, we have implicitly assumed that the entropy per atom was the same in the liquid and in the solid. This is rigorously exact only at zero temperature (however this is a good approximation up to $1.2 \mathrm{~K}$ in ${ }^{4} \mathrm{He}$ ). Therefore, at finite temperature, the atomic flow through the solid region does not preserve the lower entropy which is necessary to obtain the solid ordering. The atomic flow can achieve the required loss of entropy by emitting phonons before entering the solid region. The entropy conservation will be ensured by reabsorption of the phonons by the outcoming flow of atoms. There is a flow of mass coming through the solid region, and a distinct flow of entropy, which, instead, avoids it (Fig. 1).

Now, the solidon momentum is, approximately :

$$
\hbar K_{0}=N\left(m v+\frac{T \Delta S}{c}\right)
$$

in which $v$ is the translation velocity inside the solid region, $\Delta S$ the excess entropy per atom of the liquid and $c$ is the sound velocity. The second term in (10) represents the phonon momentum of the entropy flow. It contributes to decrease $v$, and, thus, to lower the kinetic energy of atoms inside the solid region. Eq. (4) should be replaced by

$$
E_{\mathrm{kin}}^{1}=\frac{\hbar^{2} K_{0}^{2}}{2 N m}-\frac{\hbar K_{0}}{m c} T \Delta S+\frac{1}{2} N \frac{(T \Delta S)^{2}}{m c^{2}} .
$$

The last term is only a small volume term which slightly renormalizes $\delta G$ in eq. (6) and can be neglected. Therefore, we see that the main effect of the phonon flow is a lowering of the solidon energy :

$$
\Gamma_{\mathbf{M}}^{\prime}(p, T)=\Gamma_{\mathbf{M}}(p, T)-\frac{\hbar K_{0}}{m c} T \Delta S
$$

where $\Gamma_{\mathrm{M}}(p, T)$ is given by eq. (7) or eq. (8), according as we are below or above the cross-over line defined by eq. (9).

Up to now, we have restricted this study to the solidon of smallest reciprocal lattice wave vector. Of course, solidons corresponding to larger $K$ could exist. Not too close to the melting curve, the solidon energy increase proportionally to $K$ (eq. (7)). However, since at large momenta, the phonon dispersion of the liquid increases less rapidly, the minimum might be very shallow and even not exist. This might be true also near the melting curve, where the solidon energy increases as $K^{4 / 5}$.

In the first regime $(\Gamma \sim K), n$ solidons with wave vectors $K_{0}$ have exactly the same energy as one soli- don with wave vector $n K_{0}$. Therefore, there is no tendency to solidon condensation since the condensed state has lower entropy. In the second regime $\left(\Gamma \sim K^{4 / 5}\right)$, the condensed state as a lower energy. Bound states of $n$ solidons may exist for not too large $n$. Indeed, for larger and larger $n$, the volume term in eq. (6) becomes more and more important, so that, again, $\Gamma$ is proportional to $n$ and the binding energy goes to zero.

3. Application to liquid Helium. - Although we have ignored this problem in the preceding section, the solidon is obviously strongly scattered by the individual excitations. Generally, it cannot exist as a well-defined elementary excitation with a width small compared to its energy, but rather like a fluctuation. However, we can consider two cases in which this scattering is much weaker. In the first case, the atomic masses are so heavy and the atomic velocities so small that the solidon dispersion curve is well separated in energy from the individual excitations continuum. Then, the solidon lifetime can be long. We are not aware of an experimental example in which this situation is realized. The second case is provided by the superfluid liquids in which the gap for individual excitations suppresses their scattering. Of course, superfluid ${ }^{4} \mathrm{He}$ is an ideal candidate, since it is nearly solid, as discussed above. Therefore, we are led to the conclusion that the roton minimum observed in the collective excitation dispersion curve is in fact due to solidons. One should remark that, far from its core, the solidon consists essentially in a dipolar velocity field, in close analogy with the Feynman and Cohen description. Then, one should not be surprised that the two models lead to the same qualitative predictions. A quantitative check of our theory is out of the scope of this letter, first because our calculations are very rough, and also because we lack precise data on $\delta G(p, T)$, except near the melting curve, and $\alpha$.

The value of $K_{0}$ in ${ }^{4} \mathrm{He}$ corresponds to a b.c.c. structure. This is easy to understand since, at the lowest densities, this structure has the lowest energy [8]. However, at very low temperature and near the melting curve, $\delta G$ is certainly smaller in the h.c.p. structure. But, as shown by the recent measurements of Balibar et al., the macroscopic surface tension is greater by one order of magnitude for the h.c.p. structure. It is plausible that it is also true for $\alpha$. Then, the b.c.c. solidon could be stabilized, at low pressure by $\delta G$, at high pressure by $\alpha$ (above the cross-over line).

An absolute value of the solidon gap cannot be given far from the melting curve, where $\delta G$ is badly known. Near the melting pressure, an estimate of $\alpha$ can be inferred from the positive-ion mobility measurements [10], which were interpreted by a solid-liquid surface tension coefficient $\alpha_{1 \mathrm{~s}}=0.04 \mathrm{erg} / \mathrm{cm}^{2}$. This provides an order of magnitude for the solidon gap of $7.5 \mathrm{~K}$, in very good agreement with the observed value of $7.3 \mathrm{~K}$ at 25.3 atmospheres. The correspond- 
ing number of atoms in the solid region is about 50 . The increase of the solidon gap when the pressure is lowered is in qualitative agreement with our model.

In the dipolar velocity field model, the roton gap depends on temperature because of the roton-roton interaction. Recently, Castaing and Libchaber [12] have calculated this effect by taking into account virtual exchange of phonons and of rotons between rotons (Fig. 2). In fact, such an interaction is compa-

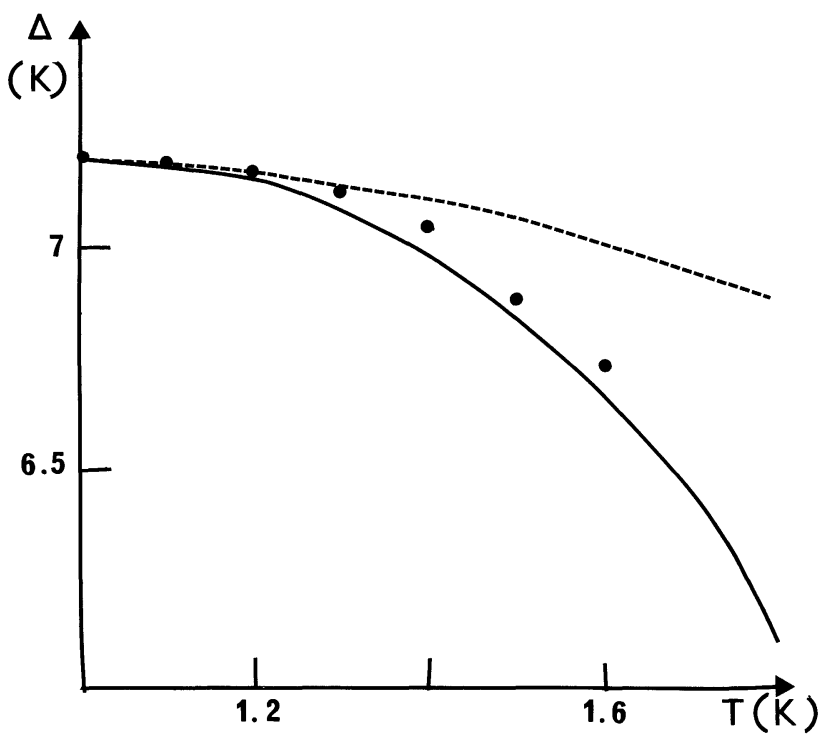

'Fig. 2. - Temperature dependence of the roton gap : - as given by the roton-roton interaction (ref. [12]) : dotted line ; - when the entropy flow also is taken into account : solid line ; - experimental points : dots.

tible with our solidon model. It would lead to temperature variation of the solidon gap. But, this gap already depends on the temperature in the independant solidon approximation, because of the entropy flow effect (eq. (12)). This can be estimated along the melting curve, where the excess entropy of the liquid is known. When the two effects are taken into account, we obtain a surprisingly good agreement with experiment (Fig. 2), if one considers the crude approximations involved.

In ${ }^{3} \mathrm{He}$, besides the zero sound branch observed at low temperature, the neutron scattering spectrum shows a broad individual excitation continuum. In this continuum, the peak of maximum intensity appears like a roton branch in the $(\omega, q)$ plane [6]. The roton minimum occurs at the expected wave vector in a b.c.c. structure. The roton gap decreases from $10 \mathrm{~K}$ at the saturated vapor pressure to less than $4 \mathrm{~K}$ at 20 bars [6]. This much greater change than in ${ }^{4} \mathrm{He}$ would indicate a smaller value of $\alpha$ and a cross-over line nearer to the melting curve. Of course, at present time, the situation is still unclear, and we would need much more precise data to draw any definite conclusion about the solidon origin of this peak.
Solidons could also exist in two dimensional nearly solid liquids. Adsorbed Helium films offer an experimental realization of these systems. Solid-liquid transitions have been seen in these films [14]. They seem to be second-order. The case of a continuous transition is particular because both $\delta G(p, T)$ and $\alpha$ should vanish at the melting curve. Therefore we expect the solidon mode to soften at the transition. Three regimes can be distinguished. In the first regime, the solidon radius is determined by $\delta G$.

Eqs. (7) and ( $\left.7^{\prime}\right)$ are still valid in two dimensions. Near the transition, at constant pressure the gap should vary as :

$$
\Gamma \sim t^{(2-\delta) / 2}
$$

where $t=\frac{T-T_{\mathrm{c}}}{T}, T_{\mathrm{c}}$ is the critical temperature and $\delta$ is the critical exponent for specific heat at constant pressure.

In the second regime, the solidon radius is determined by $\alpha$ :

$$
\Gamma \sim \alpha^{d /(2 d-1)} \sim t^{2 \sigma / 3}
$$

where $d=2$ is the space dimensionality and $\sigma$ is the critical exponent for $\alpha$.

When the melting curve is approached, one obtains, first, the one of the two regimes corresponding to the largest critical exponent, and, then, a cross-over to the other one. Finally, the third regime appears in which the solidon radius is determined by the correlation length $\xi$. In fact, the phonon frequency in the solid phase $\omega_{s}\left(K_{0}\right)$ does not vanish because of the absence of true long range order. What should vanish at the transition is :

$$
\Gamma-\omega_{\mathrm{s}}\left(K_{0}\right) \sim\left(\frac{1}{\xi}\right)^{d} \sim t^{2-\delta}
$$

since $v$, the critical exponent of $\xi$ satisfies the scaling law $\mathrm{d} v=2-\delta$.

Therefore, we expect that the critical behaviour of the solidon mode at the liquid solid transition presents, a priori, two cross-overs. Unfortunately, at present time, reliable data on the elementary excitations in liquid Helium layers are not available near the solidification, so that a comparison with the theoretical predictions is impossible.

When completing the writing of this letter, we have been aware of an abstract by O'Reilly and Tsang [13] about a New theory of superfluid ${ }^{4} \mathrm{He}$, according to which " regions of low and high mass density exist in liquid helium and moreover are spatially wellordered in the superfluid state with the result that the ground-state wave function is periodic $"$. We cannot discuss without further information the link of this new theory of superfluidity with our model of nearly solid liquid.

We acknowledge helpful discussions with W. Kohn, J. Friedel, J. M. Delrieu and M. Weger. 


\section{References}

[1] LANDAU, L. D., J.E.T.P. 11 (1941) 592.

Landau, L. D., J. Phys. Moscow 11 (1947) 91.

[2] Feynman, R. P., Statistical Mechanics, edited by J. Shaham (W. A. Benjamin) 1972.

[3] De Boer, J., Liquid Helium, editor Carreri (Academic Press) 1963.

CHester, G. V., ibid.

[4] Feymman, R. P., Phys. Rev. 94 (1954) 262.

[5] Feynman, R. P. and Cohen, M., Phys. Rev. 102 (1956) 1189.

[6] Stirling, W. G., J. Physique Colloq. 39 (1978) C6-1334.

[7] Castaing, B. and Nozières, P., J. Physique 40 (1979) 257.

[8] Hansen, J. P., J. Physique Colloq. 31 (1970) C3-67.

[9] Balibar, S., Edwards, D. O. and Laroche, C., Phys. Rev. Lett. 42 (1979) 782.
[10] Ostermeier, R. M. and Schwartz, K. W., Phys. Rev. A 5 (1972) 2510

[11] Henshaw, D. G. and Woods, A. D. G., Proc. 7th Int. Conf. Low Temp. Phys., editors Graham and Hollis Hallett (North Holland) 1961.

[12] Castaing, B. and Libchaber, A., J. Low Temp. Phys. 31 (1978) 887.

Castaing, B., Thèse d'Etat, Université Paris 6e (1978), unpublished.

[13] O'Reilly, D. E. and Tsang, T., to be published.

[14] Dash, J. G., Phys. Rep. 38C (1978) 179. 